\title{
Introduction to Automation and to its Potential for Interactive Systems Design
}

\author{
Philippe Palanque, Célia Martinie \& Elodie Bouzékri \\ * ICS-IRIT - Université Paul Sabatier Toulouse 3, Toulouse, France \\ \{palanque, martinie, bouzekri\}@irit.fr
}

\begin{abstract}
Automation is pervasive and polymorph but still usually not considered as a design option per se when designing interactive systems. This course takes a practical approach to introduce automation, its principles and how this can be used for the design of interactive systems. This one unit course introduces the automation from a Human Factors perspective (such as Levels of Automation) to highlight its foundations, its limitations and how recent research contributions demonstrate the high potential of automation as a design option for interaction and interactive systems designers. The course highlights success stories from various domains such as aviation, power plants and air traffic management. Beyond, it highlights limitations and failures from automotive, aviation (MCAS) and everyday life products (e.g. public spaces appliances). Course attendees will learn what is automation and how this high-level concept can be decomposed into practical elements that can be fruitfully used in interactive systems designs.
\end{abstract}

Keywords. Automation, Interaction Design, Interactive Systems Design, assessment of partly-autonomous systems.

\section{Introduction}

While early approaches in automation were focusing on allocating basic functions to the best player (e.g. Fitts' approach Machine Are Better At - Men Are Better At) [5], this course focuses on the use of the concept of automation for interaction design and for interactive systems designs. The course promotes the point of view of automation as a design alternative resulting on the migration of actions previously performed by users to actions performed by a system. Current push in automation is towards fully autonomous systems (such as google cars) raising critical issues such as: how to make it possible to users to foresee future states of the automation, how to disengage automation or how to make sure that users are able to take over when automation fails .... When higher automation levels are considered, users' activity gets closer to supervision, which is a different interaction paradigm. Supervision activities are very different from directly performing activities and the migration of activities to the system might have severe consequences, in terms of overall performance of the couple (user, system), in terms of skilling (and especially deskilling) [9], in terms of errors related to the very 
nature of human behavior [8], in terms of training [4]... This course promotes automation as a mean and not automation as a goal and takes a practical approach to introduce attendees to the principles, methods and tools for the design and the assessment of automation within the design of interactive systems.

\section{Contribution and benefit}

This course intends to provide newcomers with background in automation. It provides an overview on how the recent advances in automation can be exploited to design and assess interactive systems. This course will also provide material for lecturers who want to include automation in their HCI courses. Finally, the course will provide examples from various domains including aviation, Air Traffic Management, Automotive highlighting both successes and failures of automation. Concrete examples and exercises will also be provided to support the application of the course elements to practical cases.

\section{$3 \quad$ Learning Objectives}

On completion of this tutorial, attendees will:

- Know the intrinsic and extrinsic characteristics of automation, [10, 5, 2]

- Be able to use AFAR paradigm (Allocation of Functions, Authority and Responsibility) [13] when designing and assessing interactive systems,

- Know many examples on how automation has been used in various domains such as automotive, aviation, Air Traffic Control, space domain ... [11]

- Know and understand that automation is already pervasive in interactive systems and that the consequences are usually underestimated, [2]

- Have experience in analyzing an interactive systems focusing on the tasks users have to perform with it and how automation impacts user's work and activities,

- Know the danger and the benefits of more automation in interactive systems[12].

\section{Description and content}

This course focuses on the foundations of automation and how this concept can be used while designing and assessing interactive systems

- Introduction to automation its basic principles and how this concept can be decomposed in components [10] ...),

- Basic principles for describing user activity and tasks (hierarchical view on human activities, abstraction and refinement, temporal ordering, objects, information and knowledge ... [6]) and also about human activity with a focus on automation [14],

- Overview of automation designs and how they are pervasive in interactive systems and interaction techniques as well as their desired properties (intelligibility, transparency $[2], \ldots)$ 
- Overview of automation principles that are used and deployed in various domains as well as their intended objectives (enhance user, protect the user via safety or security nets, increase user comfort, entertain used, protect against automation ...).

- List of design principles for the identification of users' activities that could be good candidates for task migration towards automation, authority sharing, impact of automation degradation on tasks performance) [7].

- Taking into account human errors [1] at design time as well as possible degradation of automation [3] to assess relevance of automation in a given context (identification of types, location and likelihood of human errors)

\section{Presentation}

Lecture with slides, demonstrations and practical exercises. The lecture will include demonstration of various partly-autonomous systems that will be used to apply the principles introduced in the course. Detailed examples will be presented on large case studies in various application domains such as Air Traffic Control, Aircraft cockpits and Space Ground Segments, we will demonstrate how to deal with complex work settings.

\section{Audience and Prerequisite}

This course is open to researchers, practitioners, educators and students of all experience levels. No specific skills or knowledge are required beyond a background in User Centered Design. Paper [14] should be read before attending the lecture.

\subsection{References}

1. Fahssi R., Martinie C., Palanque P. Enhanced Task Modelling for Systematic Identification and Explicit Representation of Human Errors. IFIP INTERACT (4) 2015: LNCS, Springer Verlag 192-212.

2. Bernhaupt R., Cronel M., Manciet F., Martinie C., Palanque, P. Transparent Automation for Assessing and Designing better Interactions between Operators and Partly-Autonomous Interactive Systems. ATACCS 2015, ACM DL.

3. Fayollas C., Martinie C., Palanque P., Deleris Y., Fabre J-C., Navarre D. An Approach for Assessing the Impact of Dependability on Usability: Application to Interactive Cockpits. IEEE European Dependable Computing Conference, 2014: 198-209.

4. Martinie C, Palanque P., Navarre, D., Winckler, M., Poupart R. Model-based training: an approach supporting operability of critical interactive systems. ACM SIGCHI EICS 2011: 53-62.

5. Fitts, P.M. 1951. Human engineering for an effective air navigation and traffic control system. National Research Council, Washington, DC.

6. Martinie C., Palanque P., Ragosta M., Fahssi R. Extending procedural task models by systematic explicit integration of objects, knowledge and information. Europ. Conf. on Cognitive Ergonomics, 2013: 23-34, ACM DL. 
7. Martinie C., Palanque P., Barboni E., Ragosta M. Task-model based assessment of automation levels: Application to space ground segments. IEEE System Man and Cybernetics conference, 2011: 3267-3273.

8. Mackworth, N. H. (1948). The breakdown of vigilance during prolonged visual search. Quarterly Journal of Experimental Psychology, vol. 1, pp.6-21

9. Cavestro, W. 1990. "Beyond the Deskilling Controversy," Computer Integrated Manufacturing Systems, (3:1), pp. 38-46.

10. Palanque P. Engineering Automations: From a Human Factor Perspective to Design, Implementation and Validation Challenges. EICS 2018: 2:1-2:2

11. Meschtscherjakov A., Tscheligi M., Pfleging B., Sadeghian Borojeni S., Ju W., Palanque P., Riener A., Mutlu B., Kun A. Interacting with Autonomous Vehicles: Learning from other Domains. CHI Extended Abstracts 2018, ACM DL.

12. Palanque P., Martinie C., Fayollas C. Automation: Danger or Opportunity? Designing and Assessing Automation for Interactive Systems. Course, CHI Extended Abstracts 2018

13. Bouzekri E., Canny A., Martinie C., Palanque P., Gris C. Using Task Descriptions with Explicit Representation of Allocation of Functions, Authority and Responsibility to Design and Assess Automation. IFIP Working conf. on Human Work and Interaction Design 2018: 36-56

14. Parasuraman, R.; Sheridan, T.B. \& Wickens, C.D. 2000. A model for types and levels of human interaction with automation. Systems, Man and Cybernetics, Part A: Systems and Humans, IEEE Trans. on, vol.30, no.3, pp.286-297. 